\title{
Optimal Sensing Strategies for Mobile Robot Formations: Resource-Constrained Localization
}

\author{
Anastasios I. Mourikis and Stergios I. Roumeliotis \\ Dept. of Computer Science \& Engineering, University of Minnesota, Minneapolis, MN 55455 \\ Email: \{mourikis|stergios\}@cs.umn.edu
}

\begin{abstract}
This paper addresses the problem of resource allocation in formations of mobile robots localizing as a group. Each robot receives measurements from various sensors that provide relative (robot-to-robot) and absolute positioning information. Constraints on the sensors' bandwidth, as well as communication and processing requirements, limit the number of measurements that are available or can be processed at each time step. The localization uncertainty of the group, determined by the covariance matrix of the equivalent continuous-time system at steady state, is expressed as a function of the sensor measurements' frequencies. The trace of the submatrix corresponding to the position estimates is selected as the optimization criterion, under linear constraints on the measuring frequency of each sensor and the cumulative rate of EKF updates. This formulation leads to a convex optimization problem whose solution provides the sensing frequencies, for each sensor on every robot, required in order to maximize the positioning accuracy for the group. Simulation experiments are presented that demonstrate the applicability of this method and provide insight into the properties of the resource-constrained cooperative localization problem.
\end{abstract}

\section{INTRODUCTION}

A large number of applications require robots to move in a coordinated fashion, in order to accomplish a certain task (e.g., object moving [1], surveillance [2], platooning for efficient transportation systems [3], [4], formation flying [5], and spacecraft formations [6]). In particular, the case in which the members of a robotic team maintain constant relative positions as they traverse the space, offers certain advantages, such as simplified motion control, collision avoidance, and the ability to collectively manipulate objects in the environment. Due to the increased versatility robot formations provide, they have recently attracted significant interest in the mobile robotics community.

In this paper, we address the problem of Cooperative Localization (CL) in robot formations. Clearly, in order for a multirobot team to perform any meaningful task, it must have an estimate of the positions of its members with respect to some coordinate frame of interest. Several estimation techniques have been applied to the CL problem, such as Extended Kalman Filtering (EKF) [7], Least Squares Estimation [8], Particle Filtering [9], etc. In this work, we employ an EKF approach, similar to the one presented in [7]. The reason for this is that the EKF encompasses a well-studied mechanism, the Riccati equation, for propagating the covariance matrix of the pose estimates through time, thus providing us with a theoretically sound localization accuracy metric.

Roumeliotis and Bekey [7] have shown that proprioceptive measurements from the robots' odometry sensors can be processed locally by each robot to propagate its own pose estimates. However, every time an exteroceptive measurement is received by any of the robots in the formation, all robots must communicate their current pose estimates. Additionally, the measuring robot must transmit its new measurement in order for the EKF update to be performed. Therefore, every exteroceptive measurement that is processed incurs a penalty in terms of use of both bandwidth and CPU time. In a realistic scenario, the robots of a team will need to allocate computational and communication resources to missionspecific tasks and this may force them to reduce the number of measurements they process for localization purposes. The limitations on the available resources may thus prohibit the robots from transmitting and processing all measurements available at every time instant.

It is clear that whether or not an exteroceptive measurement should be processed in an EKF update, is determined by a tradeoff between the value of the localization information it carries, and the cost of processing it. In this paper, we assume that the robots process each of the available measurements at a constant frequency, and we seek the optimal measurement frequencies, in order to attain the highest possible positioning accuracy. The key element in our analysis is the derivation of an equivalent continuous-time system model for the robot team, whose noise parameters are functionally related to the frequency of the measurements. This enables us to express the covariance matrix of the pose errors as a functional relation of the frequencies, and thus to formulate the problem of determining the optimal sensing strategy as an optimization problem. An important result that we prove is that this problem is a convex optimization problem and therefore it is possible to find a globally optimal solution, using very efficient algorithms. Before presenting the problem formulation, in the following section we outline relevant approaches that appear in the literature.

\section{RELATED WORK}

In [10], [11], [8], localization algorithms for recovering the relative poses between the robots in a formation, using omnidirectional cameras as the primary sensors, are described. The authors propose suboptimal estimation algorithms for achieving efficient implementations. These are derived by either having each robot localize using only relative position measurements to a "leader" robot in the team, or by de- 
coupling the problems of orientation and position estimation. Both algorithms are compared to a centralized least-squares estimation algorithm, that uses all the available measurements. In presenting these methods, the trade-offs that exist between localization accuracy and the overhead for communicating and processing relative position measurements are pointed out by the authors. However, no analysis is conducted to reveal the effect of varying the available resources, and no optimal sensing strategies are proposed.

The impact of the geometry of a static robot formation on the accuracy of pose estimation is studied in the work of Zhang et al. [12]. The authors consider formations of robots that receive absolute position measurements, as well as relative measurements (i.e., relative range, bearing, or orientation). In order for the formation to be localizable, a necessary condition on the number of measurements of each type is derived. A study of the structure of the measurement equations shows that the information matrix corresponding to the exteroceptive measurements is a function of the relative positions of the robots, and a gradient-based optimization technique is employed to determine local maxima of the trace of this matrix. However, due to the non-concavity of the objective function, the selected optimization method does not guarantee global optimality of the solution. Furthermore, these results cannot be extended to the practical case of moving robots.

In [13], a robot team comprised of one master and two slave robots is studied and a portable landmarks-based technique is adopted, i.e., at each time instant at least one robot remains stationary. The robots move along a straight-line path and record measurements of their relative positions at evenly spaced intermediate points. The authors propose a method for determining the optimal relative positions between the robots and identify three configurations that yield the maximum possible localization accuracy at the end of the path. We note, however, that neither of the aforementioned approaches addresses the effects of the number and type of measurements recorded by the robots on localization accuracy. Additionally, the constraints imposed by the available computational and communication resources are not taken into consideration.

Our work is more closely related to work in the Sensor Networks community, that aims at determining the optimal scheduling of measurements, in order to attain the best possible localization of a target. Representative examples of this line of research can be found in [14], [15], [16], while a similar analysis, in the context of designing observers for dynamical systems, is presented in [17]. The defining assumption in all these cases is that a finite number of measurements is available during a certain time interval. This problem amounts to determining the optimal measurement ordering (scheduling), so as to maximize the achieved localization accuracy. For this problem, tree-search algorithms (e.g., [16]), as well as optimization methods in the continuous domain (e.g., [17]), have been proposed. This approach to the problem of finding an optimal measurement strategy is in contrast to the one employed in our work, since we here assume that the frequencies of the measurements are the design variables, and we are interested in the steady state estimation accuracy.

A different formulation of the scheduling problem has been presented in [16], [18]. In this work, the timing of each measurement is modeled by a random variable with a known probability density function (pdf). An upper bound on the $e x$ pected steady state covariance of the target's position estimate is then computed as a function of the pdf's parameters. By employing a numerical optimization routine it is possible to minimize this upper bound, and the resulting pdf is used as the optimal sensing strategy. Despite its mathematical elegance, this approach only aims at optimizing an upper bound. Since no means of determining the looseness of the bound are available, we cannot have any guarantee of optimality, or a measure of suboptimality, when this method is used.

Our work differs from the aforementioned approaches, in that we consider a team of robots that move while maintaining their formation, and localize in a global coordinate frame. The steady-state covariance matrix of the robots' localization is expressed as a function of the frequencies of all the exteroceptive measurements, and we seek to select the optimal frequencies, in order to attain the best possible positioning accuracy for the team. The constraints imposed by the available computing and communication resources are taken into account, and their effects on the accuracy of the attainable localization are examined.

\section{Problem Formulation}

We consider a team of $N$ robots that move in formation, employing a suitable control strategy in order to maintain a constant heading and constant relative positions among them. The spatial configuration of the robots is assumed to be given, defined, for example, by the application at hand. All robots are equipped with proprioceptive sensors (such as wheel encoders) that measure their translational and rotational velocities at every time step. Additionally, some (or all) of the robots are equipped with exteroceptive sensors that enable them to measure: (i) relative distance between two robots, (ii) relative bearing between two robots, (iii) absolute position of a robot, and (iv) absolute orientation of a robot. The measurements received from all the sensors are processed using an Extended Kalman Filter (EKF), in order to estimate the pose of the robots with respect to a global frame of reference.

Clearly, due to cost, reliability, or other design considerations, it may not be desirable for all robots to be equipped with identical sensors. This potential heterogeneity of the team is incorporated naturally in our approach, under the restriction that at least one robot has access, at least intermittently, to absolute position information, such as that provided by a GPS or from observing previously mapped features. This constraint is imposed because our goal is to minimize the steady-state localization uncertainty of the robots in a global coordinate frame. It is well known [19], that when no absolute position information is available to a robot team, the system is unobservable, and at steady state, the uncertainty of the robots continuously increases. The assumption for the availability of absolute positioning information could be raised if we studied 
a scenario in which only relative localization was sought. For that case, relative range and bearing measurements would (under certain conditions) be sufficient, in order to attain a bounded steady-state error covariance, and our approach would be applicable.

We now present the system and measurement models used for pose estimation.

\section{A. Propagation}

Consider $N$ non-holonomic robots moving in 2D. The discrete-time kinematic equations for the $i$-th robot are:

$$
\begin{aligned}
x_{i}(k+1) & =x_{i}(k)+V_{i}(k) \delta t \cos \left(\phi_{i}(k)\right) \\
y_{i}(k+1) & =y_{i}(k)+V_{i}(k) \delta t \sin \left(\phi_{i}(k)\right) \\
\phi_{i}(k+1) & =\phi_{i}(k)+\omega_{i}(k) \delta t, \quad i=1 \ldots N
\end{aligned}
$$

where $V_{i}(k)$ and $\omega_{i}(k)$ denote the translational and rotational velocity of the $i$-th robot at time step $k$, respectively, and $\delta t$ is the odometry sampling period. In the Kalman filter framework, the position estimates of robot $i$ are propagated using the measurements of the robot's translational and rotational velocity, $V_{m_{i}}(k)$ and $\omega_{m_{i}}(k)$, respectively. By linearizing Eqs. (1) - (3) the error propagation equation for the robot's pose is readily derived:

$$
\begin{gathered}
{\left[\begin{array}{c}
\widetilde{x}_{i_{k+1 \mid k}} \\
\widetilde{y}_{i_{k+1 \mid k}} \\
\widetilde{\phi}_{i_{k+1 \mid k}}
\end{array}\right]=\left[\begin{array}{ccc}
1 & 0 & -V_{m_{i}}(k) \delta t \sin \left(\hat{\phi}_{i}(k)\right) \\
0 & 1 & V_{m_{i}}(k) \delta t \cos \left(\hat{\phi}_{i}(k)\right) \\
0 & 0 & 1
\end{array}\right]\left[\begin{array}{c}
\widetilde{x}_{i_{k \mid k}} \\
\widetilde{y}_{i_{k \mid k}} \\
\widetilde{\phi}_{i_{k \mid k}}
\end{array}\right]} \\
+\left[\begin{array}{cc}
\delta t \cos \left(\hat{\phi}_{i}(k)\right) & 0 \\
\delta t \sin \left(\hat{\phi}_{i}(k)\right) & 0 \\
0 & \delta t
\end{array}\right]\left[\begin{array}{c}
w_{V_{i}}(k) \\
w_{\omega_{i}}(k)
\end{array}\right] \\
\Leftrightarrow \quad \widetilde{X}_{i_{k+1 \mid k}}=\Phi_{i}(k) \widetilde{X}_{i_{k \mid k}}+G_{i}(k) W_{i}(k)
\end{gathered}
$$

where $w_{V_{i}}(k)$ and $w_{\omega_{i}}(k)$ are white, zero-mean, Gaussian and uncorrelated noise sequences of variance $\sigma_{V_{i}}^{2}$ and $\sigma_{\omega_{i}}^{2}$ affecting the linear and rotational velocity measurements, respectively.

At this point, we note that since the robot team moves in a predefined formation, all robots are required to head towards the same direction, and with the same velocity, both of which are known constants. Assuming that a motion controller is used in order to minimize the deviations from the desired formation, and that the accuracy of the velocity measurements and orientation estimates is sufficiently high, we can replace the quantities $V_{m_{i}}(k), \omega_{m_{i}}(k)$, and $\hat{\phi}_{i}(k)$ in the above expressions by their respective predefined values, $V_{o}, \omega_{o}$ and $\phi_{o}$, and thus employ the approximations $\Phi_{i}(k) \simeq \Phi_{o}$ and $G_{i}(k) \simeq G_{o}$, where $\Phi_{o}$ and $G_{o}$ are the constant matrices:

$\Phi_{o}=\left[\begin{array}{ccc}1 & 0 & -V_{o} \delta t \sin \left(\phi_{o}\right) \\ 0 & 1 & V_{o} \delta t \cos \left(\phi_{o}\right) \\ 0 & 0 & 1\end{array}\right], G_{o}=\left[\begin{array}{cc}\delta t \cos \left(\phi_{o}\right) & 0 \\ \delta t \sin \left(\phi_{o}\right) & 0 \\ 0 & \delta t\end{array}\right]$

With this approximation, the state error covariance propagation equation for the $i$-th robot can be written as

$$
P_{i_{k+1 \mid k+1}}=\Phi_{o} P_{i_{k+1 \mid k}} \Phi_{o}^{T}+G_{o} Q_{i} G_{o}^{T}
$$

where $Q_{i}=\operatorname{diag}\left(\sigma_{V_{i}}^{2}, \sigma_{\omega_{i}}^{2}\right)$.
The state vector for the entire robot team is defined as the $3 N \times 1$ vector comprising of the poses $X_{i}$ of all the robots. Therefore, the covariance propagation equation for the robot formation is

$$
\mathbf{P}_{k+1 \mid k}=\boldsymbol{\Phi} \mathbf{P}_{k \mid k} \boldsymbol{\Phi}^{T}+\mathbf{Q}
$$

where $\mathbf{P}_{\ell \mid k}=\operatorname{Diag}\left(P_{i_{\ell \mid k}}\right), \boldsymbol{\Phi}=\operatorname{Diag}\left(\Phi_{o}\right)$, and $\mathbf{Q}=$ $\operatorname{Diag}\left(G_{o} Q_{i} G_{o}^{T}\right)$ are $3 N \times 3 N$ block diagonal matrices.

\section{B. Update}

The robots of the team employ the measurements recorded by their exteroceptive sensors, in order to perform pose updates in the EKF. Our method is applicable to any exteroceptive measurement model, but for simplicity, we here consider the following four types of exteroceptive measurements:

1) Relative range measurements: If robot $i$ is equipped with a sensor capable of measuring the distance of other robots with respect to itself, such as a laser scanner, then the distance measurement between robots $i$ and $j$ is

$$
z_{\rho_{i j}}(k)=\sqrt{\Delta x_{i j}(k)^{2}+\Delta y_{i j}(k)^{2}}+n_{\rho_{i j}}(k)
$$

where $\Delta x_{i j}=x_{j}-x_{i}, \Delta y_{i j}=y_{j}-y_{i}$, and $n_{\rho_{i j}}$ is a white, zero-mean, Gaussian noise process, whose standard deviation, $\sigma_{\rho_{i}}$, is determined by the characteristics of the sensor. By linearizing, the measurement error equation is derived: ${ }^{1}$

$$
\begin{aligned}
\widetilde{z}_{\rho_{i j}}(k) & =H_{\rho_{i j}}(k) \widetilde{X}(k)+n_{\rho_{i j}}(k) \\
& =\left[\begin{array}{lllllll}
0 & . . & H_{\rho_{i}} & . . & H_{\rho_{j}} & . . & 0
\end{array}\right] \widetilde{X}+n_{\rho_{i j}}
\end{aligned}
$$

where $H_{\rho_{i j}}(k)$ is a $1 \times 3 N$ matrix, whose $i$-th and $j$-th block elements are, respectively:

$$
H_{\rho_{j}}(k)=-H_{\rho_{i}}(k)=\left[\begin{array}{lll}
\widehat{\Delta x}_{i j}(k) & \widehat{\Delta y}_{i j}(k) & 0 \\
\hat{\rho}_{i j}(k) & \frac{\hat{\rho}_{i j}(k)}{} & 0
\end{array}\right]
$$

In the preceding expression, $\widehat{\Delta x}_{i j}(k), \widehat{\Delta y}_{i j}(k)$ and $\hat{\rho}_{i j}(k)$ represent the estimated differences in the $x$ and $y$ coordinates, and the estimated distance between robots $i$ and $j$, respectively. Clearly, the matrix $H_{\rho_{i j}}(k)$ is time-varying, due to its dependence on the position estimates for the robots. However, by replacing the estimates with the values corresponding to the desired formation of the robots (denoted with the subscript $o$ ), we can make the following approximations:

$$
\begin{aligned}
& H_{\rho_{i}}(k) \simeq\left[\begin{array}{lll}
\frac{-\Delta x_{i j_{o}}}{\rho_{i j_{o}}} & \frac{-\Delta y_{i j_{o}}}{\rho_{i j_{o}}} & 0
\end{array}\right]=H_{\rho_{i_{o}}} \\
& H_{\rho_{j}}(k) \simeq\left[\begin{array}{lll}
\frac{\Delta x_{i j_{o}}}{\rho_{i j_{o}}} & \frac{\Delta y_{i j_{o}}}{\rho_{i j_{o}}} & 0
\end{array}\right]=H_{\rho_{j_{o}}}
\end{aligned}
$$

Using these relations, an approximate constant value for the measurement matrix, $H_{\rho_{i j}} \simeq H_{\rho_{i j_{o}}}$ can be derived.

For practical reasons, it may not be possible for all robots to measure relative distances to all other robots in the team. For example, some robots may not be equipped with range sensors, or certain measurements may be impossible due to occlusions in the formation. In order to describe the set of all possible measurements we define the set

$$
\mathcal{H}_{\rho}=\left\{H_{\rho_{i j}} \mid \text { robot } i \text { can measure range to robot } j\right\}
$$

\footnotetext{
${ }^{1}$ To make the notation less cumbersome, in the following derivations the time step indices are omitted wherever this does not cause confusion.
} 
2) Relative bearing measurements: Assuming robot $i$ measures the relative bearing of robot $j$, the corresponding measurement equation is:

$$
z_{\theta_{i j}}(k)=\operatorname{Atan} 2\left(\Delta y_{i j}(k), \Delta x_{i j}(k)\right)-\phi_{i}(k)+n_{\theta_{i j}}(k)
$$

where $n_{\theta_{i j}}(k)$ is a white, zero-mean, Gaussian noise process, with standard deviation $\sigma_{\theta_{i}}$. Linearization yields the following measurement error equation:

$$
\begin{aligned}
\widetilde{z}_{\theta_{i j}}(k) & =H_{\theta_{i j}}(k) \widetilde{X}(k)+n_{\theta_{i j}}(k) \\
& \simeq H_{\theta_{i j_{o}}} \widetilde{X}(k)+n_{\theta_{i j}}(k) \\
& =\left[\begin{array}{lllllll}
0 & . . & H_{\theta_{i_{o}}} & . . & H_{\theta_{j_{o}}} & . . & 0
\end{array}\right] \widetilde{X}+n_{\theta_{i j}}
\end{aligned}
$$

where we have once again approximated the time-varying position estimates with their constant, desired values. Note that $H_{\theta_{i j_{o}}}$ is a $1 \times 3 N$ matrix, whose $i$-th and $j$-th block elements are, respectively:

$$
\begin{aligned}
H_{\theta_{i_{o}}} & =\left[\begin{array}{lll}
\frac{\widehat{\Delta y}_{i j_{o}}}{\hat{\rho}_{i j_{o}}^{2}} & \frac{-\widehat{\Delta x}_{i j_{o}}}{\hat{\rho}_{i j_{o}}^{2}} & -1
\end{array}\right] \\
H_{\theta_{j_{o}}} & =\left[\begin{array}{lll}
\frac{-\widehat{\Delta y_{i j_{o}}}}{\hat{\rho}_{i j_{o}}^{2}} & \frac{\widehat{\Delta x}}{\hat{\rho}_{i j_{o}}} & 0
\end{array}\right]
\end{aligned}
$$

Similarly to the case of relative range measurements, we describe all possible bearing measurements with the set

$\mathcal{H}_{\theta}=\left\{H_{\theta_{i j}} \mid\right.$ robot $i$ can measure bearing of robot $\left.j\right\}$

3) Absolute orientation measurements: Because in the EKF framework, the pose propagation equations are linearized around the current orientation estimates for each robot (cf. Eq. (4)), it is necessary to guarantee sufficiently small orientation errors for all robots. If the errors in the robots' orientation are allowed to grow unbounded, the linearization will unavoidably fail, and the EKF estimates will diverge. Therefore, it is reasonable to equip robots with absolute orientation sensors, such as, for example a compass. The measurement equation for the $i$-th robot is

$$
z_{\phi_{i}}(k)=\phi_{i}(k)+n_{\phi_{i}}(k)
$$

where $n_{\phi_{i}}$ is a white, zero-mean, Gaussian noise process, with standard deviation $\sigma_{\phi_{i}}$. The measurement error equation is:

$$
\begin{aligned}
& \widetilde{z}_{\phi_{i}}(k)=H_{\phi_{i}} \tilde{X}(k)+n_{\phi_{i}}(k) \\
& =\left[\begin{array}{lllll}
0 & . . & \underbrace{\left[\begin{array}{lll}
0 & 0 & 1
\end{array}\right]}_{i \text { th }} & . . & 0
\end{array}\right] \widetilde{X}(k)+n_{\phi_{i}}(k)
\end{aligned}
$$

All possible absolute orientation measurements are described by the set

$$
\mathcal{H}_{\phi}=\left\{\phi_{i} \mid \text { robot } i \text { can measure abs. orientation }\right\}
$$

4) Absolute position measurements: In this work, the robots localize with respect to a global coordinate frame. Therefore, in order for the position errors to remain bounded for all times, it is necessary that at least one of the robots has access to absolute position measurements. The measurement equation for the $i$-th robot is

$$
z_{p_{i}}(k)=\left[\begin{array}{ll}
x_{i}(k) & y_{i}(k)
\end{array}\right]^{T}+n_{p_{i}}(k)
$$

where $n_{p_{i}}(k)$ is a $2 \times 1$ white, zero-mean, Gaussian noise process, with covariance matrix $R_{p_{i}}$. The measurement error equation for this type of measurement is

$$
\begin{aligned}
\widetilde{z}_{p_{i}}(k) & =H_{p_{i}} \tilde{X}(k)+n_{p_{i}(k)} \\
& =\left[\begin{array}{lllll}
\mathbf{0}_{2 \times 3} & . \cdot & \underbrace{\left[\begin{array}{lll}
I_{2 \times 2} & \mathbf{0}_{2 \times 1}
\end{array}\right]}_{\text {ith block }} & & \mathbf{0}_{2 \times 3}
\end{array}\right] \widetilde{X}+n_{p_{i}}
\end{aligned}
$$

where $H_{p_{i}}$ is a $2 \times 3 N$ matrix, $I_{2 \times 2}$ denotes the $2 \times 2$ identity matrix, and $\mathbf{0}_{m \times n}$ is a $m \times n$ matrix of zeros.

In order to describe all possible absolute position measurements we define the set

$$
\mathcal{H}_{p}=\left\{H_{p_{i}} \mid \text { robot } i \text { can measure abs. position }\right\}
$$

\section{The Riccati recursion}

All exteroceptive measurements recorded by the robots at each time instant are processed by the EKF, in order to update the robots' pose estimates. The covariance update equation of the EKF is

$$
\mathbf{P}_{k+1 \mid k+1}=\mathbf{P}_{k+1 \mid k}-\mathbf{P}_{k+1 \mid k} \mathbf{H}_{k}^{T} \mathbf{S}_{k}^{-1} \mathbf{H}_{k} \mathbf{P}_{k+1 \mid k}
$$

where $\mathbf{S}_{k}=\mathbf{H}_{k} \mathbf{P}_{k+1 \mid k} \mathbf{H}_{k}^{T}+\mathbf{R}_{k}$. In these equations, $\mathbf{H}_{k}$ is the measurement matrix for the system at time step $k$, and $\mathbf{R}_{k}$ is the corresponding measurement-noise covariance matrix.

It is clear that since at each time instant a different set of measurements is recorded, $\mathbf{H}_{k}$ and $\mathbf{R}_{k}$ will not remain constant, and will possibly vary even in size at each time step. Specifically, if at time step $k$ a total of $m_{k}$ measurements are performed, $\mathbf{H}_{k}$ will comprise of $m_{k}$ block rows belonging in the set $\mathcal{H}=\mathcal{H}_{\rho} \cup \mathcal{H}_{\theta} \cup \mathcal{H}_{\phi} \cup \mathcal{H}_{p}$, and $\mathbf{R}_{k}$ will be a diagonal matrix whose elements can be defined accordingly.

Combining Eqs. (6) and (7) yields the Riccati recursion

$\mathbf{P}_{k+2 \mid k+1}=\boldsymbol{\Phi}\left(\mathbf{P}_{k+1 \mid k}-\mathbf{P}_{k+1 \mid k} \mathbf{H}_{k}^{T} \mathbf{S}_{k}^{-1} \mathbf{H}_{k} \mathbf{P}_{k+1 \mid k}\right) \boldsymbol{\Phi}^{T}+\mathbf{Q}$

that describes the discrete-time evolution of the covariance of the pose estimates for the robot team. If the system is observable, then after undergoing an initial, transient phase, the covariance matrix will enter a steady state, where its elements will fluctuate around some mean value (cf. Fig. 1). Had we been able to provide a description of this mean value as a function of the measurement frequencies, then we would have a means of directly relating the localization performance of the system to these frequencies. However, there exist no analytical tools for describing the mean value of a Riccati recursion with time-varying coefficients. To solve this problem, we propose a transition from the discrete-time system model to a continuous-time one, as described in the following section.

\section{THE RICCATI DIFFERENTIAL EQUATION}

In [20], it is shown that given a discrete-time system model, an equivalent continuous-time system model can be derived. Equivalence is established based on the requirement that the state estimates' accuracy in both systems is the same. In particular, it is shown that if state observations whose covariance is $R_{d}$ are performed at frequency $f$ in 
the discrete-time description, then the equivalent continuoustime measurements' covariance function is $E\left\{n_{c}(t) n_{c}(\tau)\right\}=$ $R_{c} \delta(t-\tau)$, where $n_{c}(\cdot)$ is the white Gaussian noise process, $\delta(\cdot)$ denotes the Dirac delta function, and $R_{c}=f^{-1} R_{d}$. The intuition behind the scaling of the covariance matrix is that it ensures a constant information influx to the continuous-time system, for any value of the sampling frequency. By a similar argument, we can derive the appropriate value of the systemnoise covariance matrix.

We now employ the idea of deriving an equivalent continuous-time system, in order to formulate a Linear Time Invariant (LTI) system model for the robot team. Specifically, since each of the measurements in the set $\mathcal{H}$ occurs at a constant frequency (generally different for each measurement), we can formulate a continuous-time system model, where all the measurements occur continuously, and the covariance of each measurement is scaled by the inverse of its frequency. In the continuous time system model, the measurement matrix $\mathbf{H}_{c}$ will be a constant matrix comprising of all the block rows in the set $\mathcal{H}$. The covariance matrix of the measurements, $\mathbf{R}_{c}$, will be a diagonal matrix, with elements the weighted covariances of the discrete-time measurements. For example, if robot $i$ receives absolute orientation measurements at a rate of $f_{\phi_{i}}$, then the continuous-time covariance corresponding to this measurement is

$$
R_{\phi_{i_{c}}}=\sigma_{\phi_{i_{c}}}^{2}=\frac{\sigma_{\phi_{i}}^{2}}{f_{\phi_{i}}}=\frac{1}{f_{\phi_{i}}} R_{\phi_{i}}
$$

We can now use the Riccati differential equation in order to describe the time evolution of the covariance of the robots' pose estimates. We note that the state transition matrix for the system in continuous time is equal to $\mathbf{F}_{c}=\operatorname{Diag}\left(F_{o}\right)$, while the matrix describing the influx of uncertainty in the continuous time system is equal to $\mathbf{Q}_{c}=\operatorname{Diag}\left(G_{o_{c}} Q_{i_{c}} G_{o_{c}}^{T}\right)$, with

$$
F_{o}=\left[\begin{array}{ccc}
0 & 0 & -V_{o} \sin \left(\phi_{o}\right) \\
0 & 0 & V_{o} \cos \left(\phi_{o}\right) \\
0 & 0 & 0
\end{array}\right], \quad G_{o_{c}}=\left[\begin{array}{cc}
\cos \left(\phi_{o}\right) & 0 \\
\sin \left(\phi_{o}\right) & 0 \\
0 & 1
\end{array}\right]
$$

and $Q_{i_{c}}=f_{o_{i}} \operatorname{diag}\left(\sigma_{V_{i}}^{2}, \sigma_{\omega_{i}}^{2}\right)$. In this last expression, $f_{o_{i}}$ denotes the rate at which robot $i$ samples its proprioceptive sensors. Using the previous relations, the Riccati differential equation is written as

$$
\dot{\mathbf{P}}(t)=\mathbf{F}_{c} \mathbf{P}(t)+\mathbf{P}(t) \mathbf{F}_{c}^{T}+\mathbf{Q}_{c}-\mathbf{P}(t) \mathbf{C P}(t)
$$

where we have defined $\mathbf{C}=\mathbf{H}_{c}^{T} \mathbf{R}_{c}^{-1} \mathbf{H}_{c}$. It is important to point out that in $\mathbf{R}_{c}^{-1}$, the frequencies of all measurements appear in the numerator of fractions on the diagonal. Therefore the elements of $\mathbf{C}$ are linear combinations of the measurement frequencies. The importance of this observation will become apparent shortly.

We note that the Riccati differential equation in Eq. (8) is a constant coefficient differential equation, and its steady state solution can be found in closed form [21]. Specifically, we define the Hamiltonian matrix

$$
\mathbf{M}_{H}=\left[\begin{array}{cc}
\mathbf{F}_{c}^{T} & -\mathbf{C} \\
-\mathbf{Q}_{c} & -\mathbf{F}_{c}
\end{array}\right]
$$

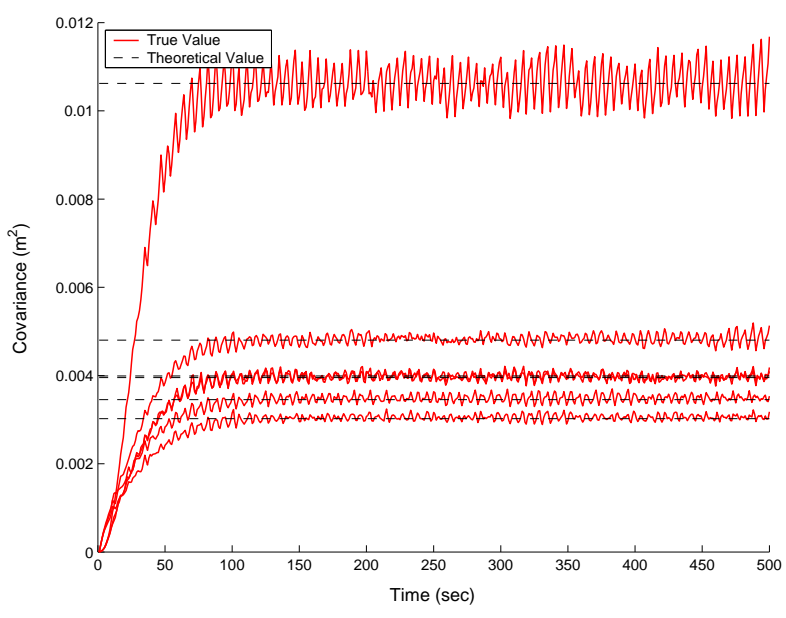

Fig. 1. True covariance vs. theoretical values. The diagonal elements of the covariance matrix corresponding to the position of the 3 robots are plotted.

and denote its eigendecomposition as $\mathbf{M}_{H}=\mathbf{U} \boldsymbol{\Lambda} \mathbf{U}^{-1}$. With this definition, the steady state solution of Eq. (8) is equal to

$$
\mathbf{P}_{s s}=\mathbf{U}_{21} \mathbf{U}_{11}^{-1}
$$

where $\mathbf{U}_{21}$ and $\mathbf{U}_{11}$ are $3 N \times 3 N$ matrices, defined by the partitioning $\mathbf{U}=\left[\mathbf{U}_{i j}\right], i, j=1,2$.

The preceding analysis shows that it is indeed possible to express the steady-state covariance of the pose estimates for the robots of the formation as a function of the measurement frequencies. To be more precise, the covariance matrix computed in Eq. (10) is the steady-state covariance of the equivalent continuous-time system, whose parameters depend on the measurement frequencies. In Fig. 1, we present the time evolution of the diagonal elements of the covariance matrix for the actual discrete-time system (solid lines) and compare them to the theoretically computed values (dashed lines) from Eq. (10). For these simulations, a team of 3 robots, that have access to all four types of exteroceptive measurements, discussed in Section III-B, was considered. The relative positions, as well as the measurement frequencies for all robots were selected randomly.

It becomes clear that, at steady state, the actual values of the covariance fluctuate around the theoretically predicted values. Thus, we can employ the continuous-time analysis in order to study the properties of the localization accuracy in the formation.

\section{Measurement Frequency Optimization}

In this section, we formulate the problem of determining the optimal measurement frequencies as a convex optimization problem. Our goal is to find the optimal frequencies for all available measurements, i.e., those frequencies that will attain the best possible localization results, under given constraints. Clearly, in order to improve the localization accuracy of the formation, the steady state covariance matrix should be minimized. However, $\mathbf{P}_{s s}$ is a $3 N \times 3 N$ matrix, and several criteria of optimality can be defined based on it (e.g., 
determinant, maximum eigenvalue, trace). A difficulty that arises is that while the elements of $\mathbf{P}_{s s}$ that correspond to the position estimates of the robots have units of $\mathrm{m}^{2}$, the elements that correspond to orientation have units of $\mathrm{rad}^{2}$. Clearly, we cannot treat these two types of elements equally. One approach would be to introduce a weight matrix $\mathbf{W}$, and try to minimize a function of the weighted matrix $\mathbf{W} \mathbf{P}_{s s} \mathbf{W}^{T}$. However, any selection of $\mathbf{W}$ would be ad-hoc and thus difficult to motivate. We have therefore chosen to focus only on the diagonal elements of $\mathbf{P}_{s s}$ that correspond to the position estimates of the robots, while making sure that the orientation uncertainty of each robot does not exceed a threshold $\epsilon_{\phi}$ (this is necessary, in order to guarantee small linearization errors). We thus formulate the following optimization problem:

$$
\begin{array}{ll}
\text { minimize } & \sum_{\substack{i=1 \\
i \neq 3 n, n \in \mathbb{N}}}^{3 N} \mathbf{P}_{s s}(i, i)+c\left(\mathbf{P}_{s s}\right) \\
\text { subject to } & 0 \leq f_{j} \leq f_{j_{\max }}, \text { for } j=1 \ldots M \\
& \sum_{j=1}^{M} f_{j}<f_{\text {total }}
\end{array}
$$

where $f_{j}$ are the frequencies of the $M$ available exteroceptive measurements, and $c\left(\mathbf{P}_{s s}\right)$ is a penalty term that is negligible whenever the orientation uncertainty of all robots is smaller than $\epsilon_{\phi}$, but becomes dominant when the threshold is exceeded. In our implementation, we have selected the function

$$
c\left(\mathbf{P}_{s s}\right)=\sum_{i=1}^{N}\left(\frac{\mathbf{P}_{s s}(3 i, 3 i)}{\epsilon_{\phi}}\right)^{100}
$$

The linear constraints on the measurement frequencies express the facts that: (i) each sensor has a maximum sampling rate, that cannot be exceeded, and (ii) the total frequency of the measurements cannot exceed a threshold, $f_{\text {total, }}$, which is determined by the available resources. We note that more general constraints can be incorporated in this formulation. For example, different types of measurements may have different costs associated with them, and this can be easily taken into consideration, by introducing weights for each of their frequencies.

In [22], it is shown that the steady-state solution of the Riccati equation in Eq. (8) is a convex function of the matrix C. Because the elements of $\mathbf{C}$ are linear functions of the measurement frequencies, we conclude that $\mathbf{P}_{s s}$ is a convex function of the measurement frequencies. As a result, the optimization problem (11) is a convex one (the objective is the sum of convex functions, and the constraints are linear). This is a very important property, because it implies that the problem does not have local minima, and we can employ standard, and very efficient, optimization algorithms for its solution. Convexity guarantees that a global optimum of the objective function will be found [23].

\section{OptimizATION RESUlTS}

This section presents simulation results that demonstrate the application of our method and provide insight into the properties of this problem. For the results shown here, a team of 4 robots, that move at $V_{o}=0.5 \mathrm{~m} / \mathrm{sec}$ in a diamond-shaped formation comprising of two adjacent equilateral triangles of side $1 \mathrm{~m}$, is considered (cf. Fig 2(a)). The robots are equipped with sensors of equal accuracy. Specifically, the standard deviation of the noise in the linear velocity measurements is equal to $\sigma_{V}=0.05 V_{o}$, while for the rotational velocity it is $\sigma_{\omega}=5 \cdot 10^{-3} \mathrm{rad} / \mathrm{sec}$. These values are consistent with the accuracy of the wheel encoders of Pioneer 1 robots, that we have determined experimentally. The standard deviation of the absolute position measurements is equal to $\sigma_{G P S}=.25 \mathrm{~m}$ along each axis, the standard deviation of the absolute orientation measurements is $\sigma_{\phi}=2^{\circ}$, while for the relative measurements between the robots we have selected $\sigma_{\rho}=0.05 \mathrm{~m}$, and $\sigma_{\theta}=3^{\circ}$. The maximum frequency for each measuring sensor is set to $f_{j_{\max }}=1 \mathrm{~Hz}, j=1 \ldots 4$. The threshold on the orientation variance for the robots, $\epsilon_{\phi}$, was selected to guarantee an orientation error standard deviation smaller than $3^{\circ}$.

In the first set of experiments, we assume that all robots can receive all types of measurements. The results of the optimization procedure, when the frequency at which measurements can be processed for the entire team is $f_{\text {total }}=2 \mathrm{~Hz}$, are shown in Table I. In the relative measurements' part of the table, the $j$-th entry in the row corresponding to robot $i\left(R_{i}\right)$, describes the frequency of the measurement performed by robot $i$, observing robot $j$. We note that approximately $65 \%$ of the measurements processed are absolute position measurements. It is interesting that at the optimal solution, no absolute orientation measurements are recorded. This implies that the correlations that exist between the position and orientation estimates of the robots suffice in order to provide sufficiently accurate orientation estimates (i.e., variance smaller than $\epsilon_{\phi}$ ) for all robots. However, it should be made clear that this is not a general result. For example, if we double the standard deviation $\sigma_{\omega}$ of the rotational velocity noise, the results of the optimization under the same conditions are shown in Table II. We note that in this case, absolute orientation measurements are processed by the robots.

In order to show the applicability of our approach to a heterogeneous robot team, we consider the case in which only one robot is equipped with a GPS receiver $\left(R_{1}\right)$. This is a realistic scenario, as cost considerations may render it impractical to equip all robots with such a device. In Table III, we show the optimization results obtained for this scenario. We notice that in this case the GPS receiver is fully utilized $\left(f_{\mathrm{GPS}_{1}}=1 \mathrm{~Hz}\right)$, since the positioning information it provides is more important than this provided by the rest of the available measurements. It is also worth noting that in this case, as in all previous ones, none of the range measurements' frequency is zero.

In Fig. 2(b), the optimal value of the cost function is plotted as a function of the total frequency of measurements $\left(f_{\text {total }}=2 . .32 \mathrm{~Hz}\right)$ for all robots in the team. We clearly observe a law of diminishing return: there is a sharp improvement in performance by increasing the total number of measurements 


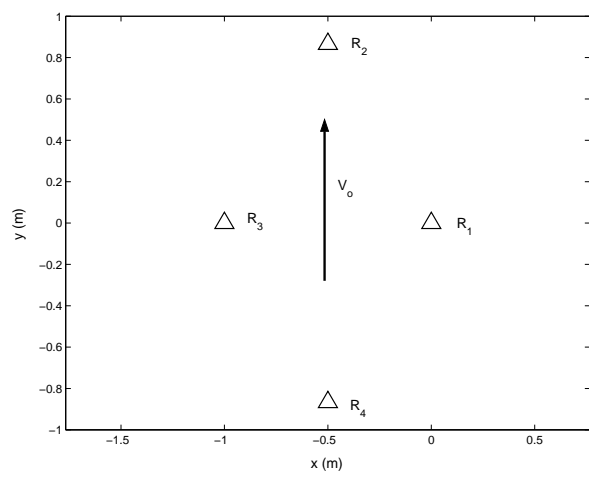

(a)

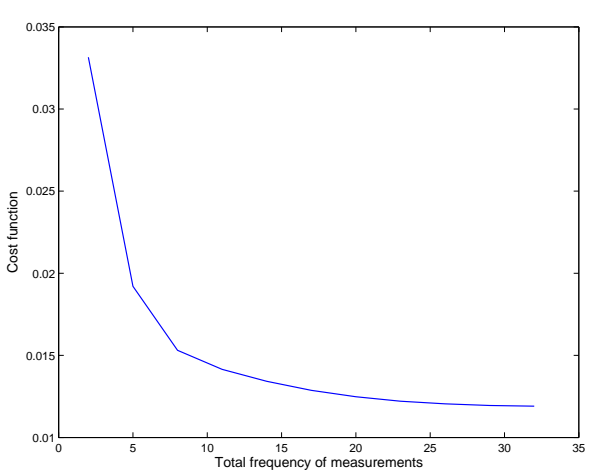

(b)

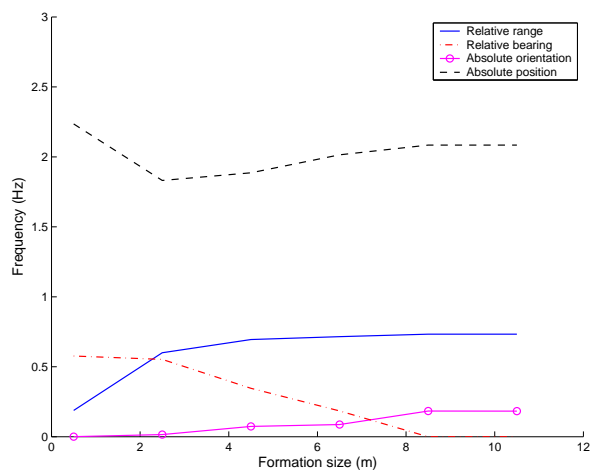

(c)

Fig. 2. (a) Robot formation and motion direction. (b) Cost function vs. Total frequency of measurements. (c) Frequency of each measurement type vs. formation size.

per time step performed by the robots, when this number is small, but the gain is lower as the number of measurements increases further. Since the necessary communication and computational resources increase linearly with the number of measurements performed by the robots, it becomes clear that unless resources are abundant, it is not beneficial for the robots to process a very large number of measurements.

In order to study the importance of processing each of the available types of measurements with varying formation size, in Fig. 2(c) we plot the total frequency of measurements assigned to each of the four possible measurement types, as a function of the length of the closest distance between any two robots in the formation. We observe that as the robots get farther from each other, the positioning information value of the relative bearing measurements diminishes, and these measurements' frequencies are equal to zero at the optimal solutions for large formation sizes. To replace the orientation information that is lost when no bearing measurements are processed, we note that the frequency of the orientation measurements increases.

As a closing remark, we note that the parameters affecting the selection of optimal measurement frequencies include the number of robots, the size and configuration of the formation in space, the robots' velocity, the accuracy of all available sensors, the type and number of available measurements and the maximum frequency of each sensor. Therefore, it is not possible, in the limited space of this paper, to demonstrate the effects of all the aforementioned parameters. The presented results are only representative.

\section{CONCLUSIONS}

In this paper we present a new approach to the resourceconstrained localization problem for formations of mobile robots. We consider heterogeneous groups of robots equipped with sensors that can provide relative and absolute positioning information at device-specific maximum frequencies. Updating the robots' position estimates requires certain measurements to be processed by an Extended Kalman Filter (EKF)

\begin{tabular}{|c|c|c|c|c|}
\hline & $R_{1}$ & $R_{2}$ & $R_{3}$ & $R_{4}$ \\
\hline & \multicolumn{4}{|c|}{$\overline{\text { Absolute position measurements }}$} \\
\hline & 0.3273 & 0.3269 & 0.0247 & 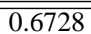 \\
\hline & \multicolumn{4}{|c|}{ Absolute orientation measurements } \\
\hline & 0 & 0 & 0 & 0 \\
\hline & \multicolumn{4}{|c|}{ Relative range measurements } \\
\hline$\overline{R_{1}}$ & $\bar{x}$ & 0.0128 & 0.0223 & 0.0109 \\
\hline$R_{2}$ & 0.0132 & $x$ & 0.0390 & 0.0501 \\
\hline$\overline{R_{3}}$ & 0.0318 & 0.0148 & $x$ & 0.0240 \\
\hline$R_{4}$ & 0.0481 & 0.0093 & 0.0175 & $\bar{x}$ \\
\hline & \multicolumn{4}{|c|}{ Relative bearing measurements } \\
\hline$R_{1}$ & $x$ & 0.0159 & 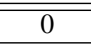 & 0.0803 \\
\hline$\overline{R_{2}}$ & 0.0159 & $\bar{x}$ & $\overline{0}$ & 0.0809 \\
\hline$R_{3}$ & 0.0808 & 0.0805 & $\bar{x}$ & $\overline{0}$ \\
\hline $\bar{R} R_{4}$ & 0 & 0 & 0 & $\bar{x}$ \\
\hline
\end{tabular}

TABLE I

Optimal Measurement Frequencies $\left(\sigma_{\omega}\right)$.

\begin{tabular}{|c|c|c|c|c|}
\hline & $R_{1}$ & $R_{2}$ & $R_{3}$ & $R_{4}$ \\
\hline & \multicolumn{4}{|c|}{ Absolute position measurements } \\
\hline & 0.2644 & 0.2629 & 0 & 0.5800 \\
\hline & \multicolumn{4}{|c|}{ Absolute orientation measurements } \\
\hline & 0.0656 & 0.0646 & 0.0323 & 0.1268 \\
\hline & \multicolumn{4}{|c|}{ Relative range measurements } \\
\hline$\overline{R_{1}}$ & $\bar{x}$ & 0.0091 & 0.0107 & 0.0271 \\
\hline$R_{2}$ & 0.0143 & $x$ & 0.0181 & 0.0330 \\
\hline $\bar{R} R_{3}$ & 0.0357 & 0.0283 & $\times$ & 0.0224 \\
\hline \multirow[t]{2}{*}{$R_{4}$} & 0.0272 & 0.0220 & 0.0112 & $\bar{x}$ \\
\hline & \multicolumn{4}{|c|}{$\begin{array}{l}\text { Relative bearing measurements } \\
\end{array}$} \\
\hline 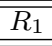 & $\times$ & 0.0130 & $\overline{0}$ & 0.0799 \\
\hline$R_{2}$ & 0.0122 & $\bar{x}$ & 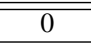 & 0.0801 \\
\hline$\overline{R_{3}}$ & 0.0798 & 0.0791 & $\bar{x}$ & $\overline{0}$ \\
\hline$R_{4}$ & 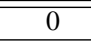 & 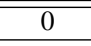 & 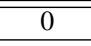 & $\bar{x}$ \\
\hline
\end{tabular}

TABLE II

Optimal Measurement Frequencies $\left(2 \sigma_{\omega}\right)$. 


\begin{tabular}{|c|c|c|c|c|}
\hline & $R_{1}$ & $R_{2}$ & $R_{3}$ & $R_{4}$ \\
\hline & \multicolumn{4}{|c|}{ Absolute position measurements } \\
\hline & 1 & 0 & 0 & 0 \\
\hline & \multicolumn{4}{|c|}{ Absolute orientation measurements } \\
\hline & 0 & 0.0431 & 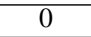 & 0.2144 \\
\hline & \multicolumn{4}{|c|}{ Relative range measurements } \\
\hline$R_{1}$ & $\times$ & 0.0615 & 0.0330 & 0.0446 \\
\hline$R_{2}$ & 0.0066 & $x$ & 0.0292 & 0.0429 \\
\hline$R_{3}$ & 0.0399 & 0.0150 & $x$ & 0.0254 \\
\hline \multirow[t]{2}{*}{$\bar{R}$} & 0.0313 & 0.0152 & 0.0132 & $x$ \\
\hline & \multicolumn{4}{|c|}{ Relative bearing measurements } \\
\hline$R_{1}$ & $\bar{x}$ & 0.0254 & 0 & 0.0896 \\
\hline$R_{2}$ & 0.0317 & $x$ & 0 & 0.0691 \\
\hline$R_{3}$ & 0.1022 & 0.0667 & $\times$ & $\overline{0}$ \\
\hline$R_{4}$ & 0 & 0 & 0 & $\times$ \\
\hline
\end{tabular}

TABLE III

Optimal MeAsurement FREQUENCIES $\left(R_{1}\right.$-GPS $)$

at rates determined by the constraints on the communication and processing resources of the team. In order to maximize the localization accuracy of the robot group, we have formulated a convex optimization problem for the minimization of the trace of the state covariance matrix. This matrix was determined as the analytical solution to the Riccati equation of the equivalent continuous-time system. The derived solutions, for different cases of sensor uncertainty, sensing frequencies, spread of the robot formation, and sensor-availability constraints were examined in simulation providing insight to the localization capabilities of different team configurations.

These results can be employed in practice for determining the sensing frequencies for robot formations of any size and shape comprised of robots with various types of sensors and sensing capabilities. The optimal sensing frequencies can be used not only for obtaining the best localization results, but also for determining the necessity of certain sensors (e.g., sensors with zero frequency can be omitted) which can lead to significant cost savings. Finally, it is our intention to capitalize on this methodology and expand our results to groups of robots that have no access to absolute position data. In this case the problem formulation will be modified so as to express the optimization criterion as a function of the covariance of the pose estimates with respect to one of the robots in the team (relative localization).

\section{ACKNOWLEDGEMENTS}

This work was supported by the University of Minnesota (DTC), the Jet Propulsion Laboratory (Grant No. 1263201), and the National Science Foundation (ITR-0324864, MRI0420836).

\section{REFERENCES}

[1] Z. Wang, Y. Hirata, and K. Kosuge, "Control a rigid caging formation for cooperative object transportation by multiple mobile robots," in Proc. of the IEEE International Conference on Robotics and Automation, New Orleans, LA, 2004, pp. 1580-1585.
[2] T. Balch and R. C. Arkin, "Behavior-based formation control for multirobot teams," IEEE Transactions on Robotics and Automation, vol. 14, no. 6, pp. 926-939, Dec. 1998.

[3] A. Broggi, M. Bertozzi, A. Fascioli, C. G. L. Bianco, and A. Piazzi, "Visual perception of obstacles and vehicles for platooning," IEEE Transactions on Intelligent Transportation Systems, vol. 1, no. 3, pp. 164-176, Sep. 2000.

[4] P. D. So and A. M. Parent, "Platooning for vehicles and automatic parking by scheduling robotic actions," IEEE Transactions on Intelligent Transportation Systems, 1996.

[5] S. Venkataramanan and A. Dogan, "Nonlinear control for reconfiguration of UAV formation," in In Proc. AIAA Guidance, Navigation, and Control Conference, Austin, TX, Aug 11-14 2003.

[6] J. Adams, A. Robertson, K. Zimmerman, and J. How, "Technologies for spacecraft formation flying," in Proc. ION-GPS 96, Sept. 17-20 1996, pp. 1321-1330.

[7] S. I. Roumeliotis and G. A. Bekey, "Distributed multirobot localization," IEEE Transactions on Robotics and Automation, vol. 18, no. 5, pp. 781795, Oct. 2002.

[8] A. Das, J. Spletzer, V. Kumar, and C. Taylor, "Ad hoc networks for localization and control," in In Proc. of the 41st IEEE Conf. on Decision and Control, Las Vegas, NV, 2002, pp. 2978- 2983.

[9] A. Howard, M. J. Mataric, and G. Sukhatme, "Putting the 'i' in 'team': an ego-centric approach to cooperative localization," in Proceedings of the IEEE International Conference on Robotics and Automation, Taipei, Taiwan, Sep. 14-19 2003, pp. 868-874.

[10] J. Spletzer, A. Das, R. Fierro, C. Taylor, V. Kumar, and J. Ostrowski, "Cooperative localization and control for multi-robot manipulation," in In Proc. of IEEE/RSJ International Conference on Intelligent Robots and Systems, Wailea, HI, 2001, pp. 631 - 636.

[11] A. Das, R. Fierro, V. Kumar, J. Ostrowski, J. Spletzer, and C. Taylor, "A vision-based formation control framework," IEEE Transactions on Robotics and Automation, vol. 18, no. 5, pp. 813 - 825, Oct. 2002.

[12] F. Zhang, B. Grocholsky, and V. Kumar, "Formations for localization of robot networks," in Proc. of the IEEE International Conference on Robotics and Automation, New Orleans, LA, 2004, pp. 3369-3374.

[13] R. Kurazume and S. Hirose, "Study on cooperative positioning system: optimum moving strategies for CPS-III." in Proceedings of the IEEE International Conference in Robotics and Automation, Leuven, Belgium, May 16-20 1998, pp. 2896-2903.

[14] D. Avintzour and S. Rogers, "Optimal measurement scheduling for prediction and estimation," IEEE Transactions on Acoustics, Speech, and Signal Processing, vol. 38, no. 10, pp. 2017-2023, October 1990.

[15] H. Lee, K. Teo, and A. E. Lim, "Sensor scheduling in continuous time," Automatica, no. 37, pp. 2017-2023, 2001.

[16] V. Gupta, T. Chung, B. Hassibi, and R. M. Murray, "Sensor scheduling algorithms requiring limited computation," in IEEE International Conference on Acoustics, Speech and Signal Processing, Montreal, Canada, May 2004, pp. 825-828.

[17] E. Skafidas and A. Nerode, "Optimal measurement scheduling in linear quadratic gaussian control problems," in Proceedings of the IEEE International Conference on Control Applications, Trieste, Italy, Sept. 1998, pp. 1225 - 1229.

[18] T. H. Chung, V. Gupta, B. Hassibi, J. W. Burdick, and R. M. Murray, "Scheduling for Distributed Sensor Networks with Single Sensor Measurement Per Time Step," in Proc. of IEEE Conf. on Robotics and Automation, New Orleans, LA, April 2004, pp. 187-192.

[19] A. I. Mourikis and S. I. Roumeliotis, "Analysis of Positioning Uncertainty in Reconfigurable Networks of Heterogeneous Mobile Robots," in Proceedings of the 2004 IEEE International Conference on Robotics and Automation, New Orleans, LA, USA, Apr. 26 - May 1 2004, pp. 572-579.

[20] P. S. Maybeck, Stochastic Models, Estimation, and Control. Academic Press, 1979, vol. 141-1.

[21] W. L. Brogan, Modern Control Theory. Prentice Hall, 1991.

[22] G. Freiling and V. Ionescu, "Monotonicity and convexity properties of matrix Riccati equations," IMA Journal of Mathematical Control and Information, no. 18, pp. 61-72, 2001.

[23] S. Boyd and L. Vandenberghe, Convex Optimization. Cambridge University Press, 2004. 